\title{
PAC LEARNING, VC DIMENSION, AND THE ARITHMETIC HIERARCHY
}

\author{
WESLEY CALVERT
}

\begin{abstract}
We compute that the index set of PAC-learnable concept classes is $m$-complete $\Sigma_{3}^{0}$ within the set of indices for all concept classes of a reasonable form. All concept classes considered are computable enumerations of computable $\Pi_{1}^{0}$ classes, in a sense made precise here. This family of concept classes is sufficient to cover all standard examples, and also has the property that PAC learnability is equivalent to finite $\mathrm{VC}$ dimension.
\end{abstract}

\section{INTRODUCTION}

A common method to characterize the complexity of an object is to describe the degree of its index set [5, 6, 7, 8, 10, 13, 16. In the present paper, we carry out this computation for the class of objects which are machine-learnable in a particular model.

There have been several models of machine learning, dating back at least to Gold's seminal 1967 paper 12. In Gold's basic model, the goal is that the machine should determine a $\Sigma_{1}^{0}$-index for a computably enumerable set of natural numbers - that is, an index for a computable function enumerating it, by receiving an initial segment of the string. Of course, many variations are possible, involving, for instance, the receipt of positive or negative information and the strength of the convergence criteria in the task of "determining" an index. This family of models has been studied by the recursion theory community (see, for instance, [11, 14, 19]), but is not the primary focus of this paper. One particular result, however, is of interest to us.

Theorem 1.1 (Beros [1]). The set of $\Sigma_{1}^{0}$ indices for uniformly computably enumerable families learnable in each of the following models is $m$-complete in the corresponding class.

(1) TxtFin $-\Sigma_{3}^{0}$

(2) $T x t E x-\Sigma_{4}^{0}$

(3) $T x t B C-\Sigma_{5}^{0}$

(4) $T x t E x^{*}-\Sigma_{5}^{0}$

1.1. PAC Learning. The model of learning that concerns us here (PAC learning, for "Probably Approximately Correct") was first proposed by Valiant in [20. Much of our exposition of the subject comes from [15. The idea of the model is that it should allow some acceptably small error of each of two kinds: one arising from targets to be learned which are somehow too close together to be easily distinguished, and the other arising from randomness in the examples shown to the learner. Neither aspect is easily treated in Gold's framework of identifying indices

Date: September 4, 2018. 
for computable enumerations of natural numbers by inspecting initial segments neither a notion of "close" nor randomness in the inputs.

In the present paper, we will describe a framework in which to model PAC learning in a way which is suitable for recursion-theoretic analysis and which is broad enough to include many of the benchmark examples. We will then calculate the $m$-degree of the set of indices for learnable concept classes.

Definition 1.2 (Valiant).

(1) Let $X$ be a set, called the instance space.

(2) Let $\mathcal{C}$ be a subset of $\mathcal{P}(X)$, called a concept class.

(3) The elements of $\mathcal{C}$ are called concepts.

(4) We say that $\mathcal{C}$ is $P A C$ Learnable if and only if there is an algorithm $\varphi_{e}$ such that for every $c \in \mathcal{C}$, every $\epsilon, \delta \in\left(0, \frac{1}{2}\right)$ and every probability distribution $\mathcal{D}$ on $X$, the algorithm $\varphi_{e}$ behaves as follows: On input $(\epsilon, \delta)$, the algorithm $\varphi_{e}$ will ask for some number $n$ of examples, and will be given $\left\{\left(x_{1}, i_{1}\right), \ldots,\left(x_{n}, i_{n}\right)\right\}$ where $x_{j}$ are independently randomly drawn from $\mathcal{D}$, and $i_{j}=\chi_{c}\left(x_{j}\right)$. The algorithm will then output some $h \in \mathcal{C}$ so that with probability at least $1-\delta$ in $\mathcal{D}$, the symmetric difference of $h$ and $c$ has probability at most $\epsilon$ in $\mathcal{D}$.

This is a well-studied model - so well-studied, in fact, that it is more usual to talk about the complexity of the algorithm (in both running time and the number of example calls) than about its existence. For the present paper, though, we restrict ourselves to the latter problem. Several examples are well-known.

Example 1.3. Let $X=2^{n}$, interpreted as assignments of truth values to Boolean variables. Then the class $\mathcal{C}$ of $k$-CNF expressions is PAC learnable (where each expression $c \in \mathcal{C}$ is interpreted as the set of truth assignments that satisfy it).

Example 1.4. Let $X=\mathbb{R}^{d}$. Then the class $\mathcal{C}$ of linear half-spaces is PAC learnable.

Example 1.5. Let $X=\mathbb{R}^{2}$. Then the class of convex $d$-gons is PAC learnable for any $d$.

1.2. The Vapnik-Chervonenkis Dimension. An alternate view of PAC learnability arises from work of Vapnik and Chervonenkis 21. Again, we follow the exposition of [15].

Definition 1.6. Let $\mathcal{C}$ be a concept class.

(1) Let $S \subseteq X$. Then $\Pi_{\mathcal{C}}(S)=|\{S \cap c: c \in \mathcal{C}\}|$.

(2) The VC dimension of $\mathcal{C}$ is the greatest integer $d$ such that $\Pi_{\mathcal{C}}(S)=2^{d}$ for some $S$ with cardinality $d$, if such an integer exists. Otherwise, the VC dimension of $\mathcal{C}$ is $\infty$.

For example, if $\mathcal{C}$ is the class of linear half-spaces of $\mathbb{R}^{2}$, and if $S$ is a set of size 4 , suppose that $k$ is the least such that all of $S$ is contained in the convex hull of $k \leq 4$ points. If $k<4$, take a set $S_{0}$ of size $k$ such that the convex hull of $S_{0}$ contains $S$. The subset $S_{0} \subset S$ cannot be defined by intersecting $S$ with a linear half-space. If $k=4$, then let $S_{0}$ be a diagonal pair, which again cannot be defined by intersection with a linear half-space. Consequently, the VC dimension of $\mathcal{C}$ must be at most 3 . One can also show that this bound is sharp. 
The connection of $\mathrm{VC}$ dimension with learnability is a theorem of Blumer, Ehrenfeucht, Haussler, and Warmuth showing that under some reasonable measuretheoretic hypotheses (which hold in all examples shown so far, and in all examples that will arise in the present paper), finite $\mathrm{VC}$ dimension is equivalent to PAC learnability 3 .

Definition 1.7 (Ben-David, as described in [3]). Let $R \subseteq \mathcal{P}(X)$, and let $\mathcal{D}$ be a probability distribution on $X$, and $\epsilon>0$.

(1) We say that $N \subseteq X$ is an $\epsilon$-transversal for $R$ with respect to $\mathcal{D}$ if and only if for any $c \in R$ with $P_{\mathcal{D}}(c)>\epsilon$ we have $N \cap R \neq \emptyset$.

(2) For each $m \geq 1$, we denote by $Q_{\epsilon}^{m}(R)$ the set of $\vec{x} \in X^{m}$ such that the set of distinct elements of $\vec{x}$ does not form an $\epsilon$-transversal for $R$ with respect to $\mathcal{D}$.

(3) For each $m \geq 1$, we denote by $J_{\epsilon}^{2 m}(R)$ the set of all $\vec{x} \vec{y} \in X^{2 m}$ with $\vec{x}$ and $\vec{y}$ each of length $m$ such that there is $c \in R$ with $P_{\mathcal{D}}(c)>\epsilon$ such that no element of $c$ occurs in $\vec{x}$, but elements of $c$ have density at least $\frac{\epsilon m}{2}$ in $\vec{y}$.

(4) We say that a concept class $\mathcal{C}$ is well-behaved if for every Borel set $b$, the sets $Q_{\epsilon}^{m}(R)$ and $J_{\epsilon}^{2 m}(R)$ are measurable where $R=\{c \triangle b: c \in \mathcal{C}\}$.

This notion of "well-behaved" is exactly the necessary hypothesis for the equivalence:

Theorem $1.8([3)$. Let $\mathcal{C}$ be a nontrivial, well-behaved concept class. Then $\mathcal{C}$ is $P A C$ learnable if and only if $\mathcal{C}$ has finite $V C$ dimension.

\section{Concepts and Concept Classes}

The most general context in which PAC learning makes sense is far too broad to say anything meaningful about the full problem of determining whether a class is learnable. If we were to allow the instance space to be an arbitrary set, and a concept class an arbitrary subset of the powerset of the instance space, we would quickly be thinking about a non-trivial fragment of set theory.

In practice, on the other hand, one usually fixes the instance space, and asks whether (or how efficiently, or just by what means) a particular class is learnable. This approach is too narrow for the main problem of this paper to be meaningful. The goal of this section, then, is to describe a context broad enough to cover many of the usual examples, but constrained enough to be tractable.

Many of the usual examples of machine learning problems can be systematized in the framework of $\Pi_{1}^{0}$ classes, which will now be introduced. The following result is well-known, but a proof is given in [9], which is also a good general reference on $\Pi_{1}^{0}$ classes.

Theorem 2.1. Let $c \subseteq 2^{\omega}$. Then the following are equivalent:

(1) $c$ is the set of all infinite paths through a computable subtree of $2^{\omega}$

(2) $c$ is the set of all infinite paths through a $\Pi_{1}^{0}$ subtree of $2^{\omega}$ (i.e. a co-c.e. subtree)

(3) $c=\left\{x \in 2^{\omega}: \forall n R(n, x)\right\}$ for some computable relation $R$, i.e. a relation $R$ for which there is a Turing functional $\Phi$ such that $R(n, x)$ is defined by $\Phi^{x}(n)$.

This equivalence (and other similar formulations could be added) gives rise to the following definition: 
Definition 2.2. Let $c \subseteq 2^{\omega}$. Then we say that $c$ is a $\Pi_{1}^{0}$ class if and only if it satisfies one of the equivalent conditions in Theorem 2.1.

Example 2.3. There is a natural and uniform representation of all well-formed formulas of classical propositional calculus, each as a $\Pi_{1}^{0}$ class. We regard $2^{\omega}$ as the assignment of values to Boolean variables, so that for $f \in 2^{\omega}$, the value $f(n)=k$ indicates a value of $k$ for variable $x_{n}$. Let $\varphi$ be a propositional formula. We construct a $\Pi_{1}^{0}$ subtree $T_{\varphi} \subseteq 2^{\omega}$ such that $f \in T_{\varphi}$ if and only if $f$ satisfies $\varphi$. At stage $n$, for each $\sigma \in 2^{<\omega}$ of length $n$, we include $\sigma \in T_{\varphi}$ if and only if there is an extension $f \supset \sigma$ such that $F \models \varphi$. This condition can be checked effectively. Consequently, $T_{\varphi}$ is a $\Pi_{1}^{0}$ subtree of $2^{\omega}$ — intuitively, an infinite path $f$ may fall out of $T_{\varphi}$ at some point when we see a long enough initial segment to detect nonsatisfiability, but unless it falls out at some finite stage, it is included.

Example 2.4. There is a natural and uniform representation of all closed intervals of $\mathbb{R}$ with computable endpoints, each as a $\Pi_{1}^{0}$ class. We take the usual representation of real numbers by binary strings. Let $I$ be a closed interval with computable endpoints. We construct a $\Pi_{1}^{0}$ tree $T_{I} \subseteq 2^{\omega}$ such that the set of paths through $T_{I}$ is equal to $I$. At stage $s$, we include in $T_{I}$ all binary sequences $\sigma$ of length $s$ such that there is an extension $f \supset \sigma$ with $f \in I$. This condition can be checked effectively, by the computability of the endpoints of $I$. Consequently, $T_{I}$ is a $\Pi_{1}^{0}$ subtree of $2^{\omega}$.

Example 2.5. There is a natural and uniform representation of all closed linear half-spaces of $\mathbb{R}^{d}$ which are defined by hyperplanes with computable coefficients, each half-space as a $\Pi_{1}^{0}$ class. We encode $\mathbb{R}^{d}$ as $2^{\omega}$ in the following way: the $i$ th coordinate of the point represented by the path $f$ is given by the sequence $(f(k): k \equiv i \bmod d)$. Now we encode a linear subspace into a subtree in the same way as with intervals in the previous example.

Example 2.6. There is a natural and uniform representation of all convex d-gons in $\mathbb{R}^{2}$ with computable vertices, with each $d$-gon represented by a $\Pi_{1}^{0}$ class. A convex $d$-gon is an intersection of $d$ closed linear half-spaces, and so we exclude a node $\sigma \in 2^{\omega}$ from the tree for our $d$-gon if and only if it is excluded from the tree for at least one of those linear half-spaces.

Note that the requirement of computable boundaries of these examples is not a practical restriction.

Proposition 2.7. For any probability measure $\mu$ on $\mathbb{R}^{d}$ absolutely continuous with respect to Lebesgue measure, and for any hyperplane given by $f(\vec{x})=0$, there is a hyperplane given by $\bar{f}(\vec{x})=0$ where $\bar{f}$ has computable coefficients, and where the linear half-spaces defined by these hyperplanes are close in the following sense: If $H_{f}$ is defined by $f(\vec{x}) \leq 0$, if $H_{f}^{0}$ is defined by $f(\vec{x})<0$, and $H_{\bar{f}}$ is defined by $\bar{f}(\vec{x}) \leq 0$, then $\mu\left(H_{f} \triangle H_{\bar{f}}\right)<\epsilon$ and $\mu\left(H_{f}^{0} \triangle H_{\bar{f}}\right)<\epsilon$.

Proof. Since a hyperplane has Lebesgue measure 0, it suffices to show that we can achieve $\mu\left(H_{f} \triangle H_{\bar{f}}\right)<\epsilon$. Now by using the cumulative distribution function, we can construct a bounded $d$-orthotope $B \subseteq \mathbb{R}^{d}$ with computable vertices such that $\mu(B) \geq \frac{\epsilon}{2}$.

Since computable points are dense in $\mathbb{R}$, we can find, in each face $F_{i}$ of $B$, a computable point $\vec{a}_{i}$ so close to $f(\vec{x})=0$ that if $\vec{f}(\vec{x})=0$ is the hyperplane 
determined by the set of points $\left\{\vec{a}_{i}: i \leq d\right\}$, then

$$
\mu\left(\left(H_{f} \triangle H_{\bar{f}}\right) \cap B\right)<\frac{\epsilon}{2} .
$$

The coefficients of $\bar{f}$ are computable since the points $\vec{a}_{i}$ are computable. Furthermore,

$$
\mu\left(H_{f} \triangle H_{\bar{f}}\right)<\epsilon .
$$

Examples could be multiplied, of course, and it seems likely that many of the more frequently encountered machine learning situations could be included in this framework - certainly, for instance, any example in [2], [15], or [17.

We will work, for the purposes of the present paper, with instance space $2^{\omega}$ and with concepts which are $\Pi_{1}^{0}$ classes. It remains to describe the concept classes to be used.

There is an unfortunate clash of terminology in that the concept classes will have, for their members, $\Pi_{1}^{0}$ classes. In this paper, we will never use the term ambiguously, but because both terms are so well-established it will be necessary to use both of them.

Definition 2.8. A weakly effective concept class is a computable enumeration $\varphi_{e}: \mathbb{N} \rightarrow \mathbb{N}$ such that $\varphi_{e}(n)$ is a $\Pi_{1}^{0}$ index for a $\Pi_{1}^{0}$ tree $T_{e, n}$.

Naturally, we interpret each index enumerated as the $\Pi_{1}^{0}$ class of paths through the associated tree. We also freely refer to the indices (or trees, or $\Pi_{1}^{0}$ classes) in the range of a concept class as its elements.

This definition is almost adequate to our needs. We would like, however, one additional property: that a finite part of an effective concept class should not be able to distinguish a non-computable point of $2^{\omega}$ from all computable points. This is reasonable: it would strain our notion of an "effective" concept class if it should fail. And yet it can fail with a weakly effective concept class: our classes may have no computable members at all, for instance. For that reason, we define an effective concept class as follows.

Definition 2.9. An effective concept class is a weakly effective concept class $\varphi_{e}$ such that for each $n$, the set $c_{n}$ of paths through $T_{e, n}$ is computable in the sense that there is a computable function $f_{c_{n}}(d, r): 2^{<\omega} \times \mathbb{Q} \rightarrow\{0,1\}$ such that

$$
f_{c_{n}}(\sigma, r)= \begin{cases}1 & \text { if } B_{r}(\sigma) \cap c_{n} \neq \emptyset \\ 0 & \text { if } B_{2 r}(\sigma) \cap c_{n}=\emptyset \\ 0 \text { or } 1 & \text { otherwise }\end{cases}
$$

where $B_{r}(\sigma)$ is the set of all paths that either extend $\sigma$ or first differ from it at the $-\lceil l g(r)\rceil$ place or later (see [4, 22]).

In addition to the useful property mentioned above, which we will soon prove, there is another reason for preferring this stronger definition: Typically when we want a computer to learn something, it is with the goal that the computer will then be able to act on it. Computability of each concept is a necessary condition for this. The restriction corresponds, in the examples, to the restriction that a linear half-space, for instance, be defined by computable coefficients. The classes we consider in this paper will be effective concept classes. 
Proposition 2.10. Let $\mathcal{C}$ be an effective concept class, and let $c_{1}, \ldots, c_{k} \in \mathcal{C}$. Then for any $y \in 2^{\omega}$, there is a computable $x \in 2^{\omega}$ such that for each $i \in\{1, \ldots, k\}$, we have $x \in c_{i}$ if and only if $y \in c_{i}$.

Proof. Let $y, c_{1}, \ldots, c_{k}$ be as described in the statement of the Proposition. Let $I$ be the set of $i$ such that $y \in c_{i}$ and $J$ be the set of $i$ such that $y \notin c_{i}$.

Suppose first that $y$ is not in the boundary $\partial c_{i}$ of $c_{i}$ for each $i$. Then

$$
y \in N:=\left(\bigcap_{i \in I} c_{i}^{\circ}\right) \cap\left(\bigcap_{i \in J}\left(\overline{c_{i}}\right)^{\circ}\right),
$$

where $S^{\circ}$ denotes the interior of $s$ and $\bar{S}$ the complement of $S$. Since $I$ and $J$ are finite, $N$ is open. Since $y \in N$, the set $N$ is nonempty, and must contain a basic open set of $2^{\omega}$, and so must contain a computable member, $x$, as required.

Now suppose that $y$ is in $\partial c_{i}$ for some $i$. Then we can compute $y$, using the function $f_{c_{i}}$, so that $y$ is itself computable and we take $x=y$.

We note that all of the examples given so far are effective concept classes.

Example 2.11. The class of well-formed formulas of classical propositional calculus, and the class of $k$-CNF expressions (for any $k$ ) are effective concept classes, by the example above. Whether a given $y \in 2^{\omega}$ satisfies a particular formula can be determined by examining only finitely many terms of $y$.

Example 2.12. The class $\mathcal{C}$ of linear half-spaces in $\mathbb{R}^{d}$ bounded by hyperplanes with computable coefficients is an effective concept class. Recall that each linear half-space with computable coefficients is a computable set, since the distance of a point from the boundary can be computed.

Example 2.13. The class of convex $d$-gons in $\mathbb{R}^{2}$ with computable vertices is an effective concept class.

Again, it appears that any example in any of the standard references is an effective concept class.

A pleasant feature of the effective concept classes is that they are always wellbehaved.

Lemma 2.14. A weakly effective concept class has finite $V C$ dimension if and only if it is PAC learnable.

Proof. Let $\mathcal{C}$ be an effective concept class. In [3], a proof of Ben-David is given that if $\mathcal{C}$ is universally separable - that is, if there is a countable subset $\mathcal{C}^{*}$ such that every point in $\mathcal{C}$ can be written as the pointwise limit of some sequence in $\mathcal{C}^{*}-$ then $\mathcal{C}$ is well-behaved. Since an effective concept class is always countable (i.e. it contains only countably many $\Pi_{1}^{0}$ classes $), \mathcal{C}$ is trivially universally separable. By Theorem 1.8, the conclusion holds.

\section{Bounding the Degree of the Index Set}

We now turn toward the main problem of the paper, which we can now express exactly.

Problem 3.1. Determine the $m$-degree of the set of all natural numbers $e$ such that $\varphi_{e}$ is a PAC-learnable effective concept class. 
One minor refinement in the problem remains: the difficulty of saying that $e$ is the index for an effective concept class competes with that of saying that this concept class is learnable. Indeed, since determining that $n$ is an $X$-index for an $X$-computable tree is $m$-complete $\Pi_{2}^{0}(X)$ (see $[13,18$ ), it follows that determining that $n$ is a $\Pi_{1}^{0}$ index for a $\Pi_{1}^{0}$ tree is $m$-complete $\Pi_{3}^{0}$.

Since we will see that finite VC dimension can be defined at $\Sigma_{3}^{0}$, a driving force in the $m$-degree described in the problem above will be that it must compute all $\Pi_{3}^{0}$ sets. This tells us nothing about the complexity of learnability, but only about the complexity of determining whether we have a concept class. The usual way to deal with this issue is by the following definition.

Definition $3.2\left([5)\right.$. Let $A \subseteq B$, and let $\Gamma$ be some class of sets (e.g. $\left.\Pi_{3}^{0}\right)$.

(1) We say that $A$ is $\Gamma$ within $B$ if and only if $A=R \cap B$ for some $R \in \Gamma$.

(2) We say that $S \leq_{m} A$ within $B$ if and only if there is a computable $f: \omega \rightarrow B$ such that for all $n$ we have $n \in S \Leftrightarrow f(n) \in A$.

(3) We say that $A$ is $m$-complete $\Gamma$ within $B$ if and only if $A$ is $\Gamma$ within $B$ and for every $S \in \Gamma$ we have $S \leq_{m} A$ within $B$.

We can now present the question in its final form.

Problem 3.3. Let $L$ be the set of indices for effective concept classes, $K$ the set of indices for effective concept classes which are PAC learnable. What is the $m$-degree of $K$ within $L$ ?

The solution to the problem will have two parts. In the present section, we will show that $K$ is $\Sigma_{3}^{0}$ within $L$. In the following section, we show that $K$ is $m$-complete $\Sigma_{3}^{0}$ within $L$.

We first reduce the problem to one on computable paths through $2^{\omega}$.

Proposition 3.4. An effective concept class $\mathcal{C}$ has infinite $V C$ dimension if and only if for every d there are (not necessarily uniformly) computable elements

$$
\left(x_{i}: i<d\right)
$$

such that $\Pi_{\mathcal{C}}\left(x_{i}: i<d\right)=2^{d}$..

Proof. Let $\left(y_{i}: i<d\right)$ witness that $\mathcal{C}$ has VC dimension at least $d$, and denote by $D_{1}, \ldots D_{2^{d}}$ elements of $\mathcal{C}$ which distinguish distinct subsets of $\left(y_{i}: i<d\right)$. For each $i<d$, there is a computable element $x_{i}$ such that for every $j \leq 2^{d}$ we have $x_{i} \in D_{j}$ if and only if $y_{i} \in D_{j}$, by Proposition 2.10. Then $x_{1}, \ldots x_{d}$ witness that $\mathcal{C}$ has $\mathrm{VC}$ dimension at least $d$. The converse is obvious.

Proposition 3.5. The set of indices for effective concept classes of infinite $V C$ dimension is $\Pi_{3}^{0}$ within $L$.

Proof. We begin by noting that if $f$ is a computable function and $T$ is a $\Pi_{1}^{0}$ tree, then it is a $\Pi_{1}^{0}$ condition that $f$ is a path of $T$, and a $\Sigma_{1}^{0}$ condition that it is not, uniformly in a $\Pi_{1}^{0}$ index for $T$ and a computable index for $f$. Further, if $\mathcal{C}=\varphi_{e}$ is an effective concept class, then for any $k \in \omega$, the condition that $k \in \operatorname{ran}\left(\varphi_{e}\right)$ is a $\Sigma_{1}^{0}$ condition, uniformly in $e$ and $k$.

Let $\left(x_{1}, \ldots, x_{n}\right)$ be a sequence of computable functions, $S \subseteq\{1,2, \ldots, n\}$, and $c$ a $\Pi_{1}^{0}$ class, represented by a $\Pi_{1}^{0}$ index for a tree in which it is the set of paths. We abbreviate by $c\left\lceil_{n}=S\right.$ the statement that for each $i \in\{1, \ldots n\}$, we have $x_{i} \in c$ if 
and only if $i \in S$. Now $c \uparrow_{n}=S$ is a $d$ - $\Sigma_{1}^{0}$ condition, uniformly in the indices for the $x_{i}$ and $c$.

We now note that $\mathcal{C}=\varphi_{e}$ has infinite $\mathrm{VC}$ dimension if and only if

$$
\bigwedge_{n \in \mathbb{N}} \exists x_{1}, \ldots, x_{n} \bigwedge_{S \subseteq(n+1)} \exists k\left[\varphi_{e}(k) \uparrow_{n}=S\right]
$$

From the comments above, this definition is $\Pi_{3}^{0}$.

\section{Sharpness of the Bound}

The completeness result in this section will finish our answer to the main question of the paper.

Theorem 4.1. The set of indices for effective concept classes of infinite $V C$ dimension is $m$-complete $\Pi_{3}^{0}$ within $L$, and the set of indices for effective concept classes of finite $V C$ dimension is $\Sigma_{3}^{0}$ within $L$.

Proof. It only remains to show completeness. For each $\Pi_{3}^{0}$ set $S$, we will construct a sequence of effective concept classes $\left(\mathcal{C}_{n}: n \in \mathbb{N}\right)$ such that $\mathcal{C}_{n}$ has infinite VC dimension if and only if $n \in S$. In the following lemma, to simplify notation, we suppress the dependence of $f$ on $n$.

Lemma 4.2. There is a $\Delta_{2}^{0}$ function $f: \mathbb{N} \rightarrow 2$ such that $f(s)=1$ for infinitely many $s$ if and only if $n \in S$.

Proof. It suffices (see [18]) to consider $S$ of the form $\exists^{\infty} x \forall y R(x, y, n)$. Now we set

$$
f(x)=\left\{\begin{array}{ll}
1 & \text { if } \forall y R(x, y, n) \\
0 & \text { otherwise }
\end{array} .\right.
$$

This function is $\Delta_{2}^{0}$-computable, and has the necessary properties.

Now by the Limit Lemma, there is a uniformly computable sequence $\left(f_{s}: s \in \mathbb{N}\right)$ of functions such that for each $x$, for sufficiently large $s$, we have $f_{s}(x)=f(x)$.

We now take a set of functions that will serve as the elements that may eventually witness high $\mathrm{VC}$ dimension. Let $\left\{\pi_{s, t, j}: s, t, j \in \mathbb{N}, j<s\right\}$ be a discrete uniformly computable set of distinct elements of $2^{\omega}$ such that $\pi_{s, t, j}(q)=\pi_{s, t^{\prime}, j^{\prime}}(q)$ whenever $q<\min \left\{t, t^{\prime}\right\}$.

We also initialize $G_{s, 0}=\emptyset$ for each $s$. Denote by $P_{t}$ a bijection

$$
P_{t}: \mathcal{P}(\{1, \ldots, t\}) \rightarrow\left\{1, \ldots, 2^{t}\right\} .
$$

At stage $s$ of the construction, we consider $f_{s}(t)$ for each $t \leq s$. If $f_{s}(t)=0$, then no action is required.

If $f_{s}(t)=1$, then we find the least $k$ such that $k \notin G_{t, s}$. Let $\left\{e_{t, i}: i<2^{t}\right\}$ be $\Pi_{1}^{0}$ indices for trees such that $T_{e_{t, i}}$ consists exactly of the initial segments $\tau$ of $\pi_{t, k, j}$ where $j=P_{t}(S)$ for some $S \subseteq\{1, \ldots, t\}$ and $|\tau|$ is less than the first $z>s$ such that $f_{z}(t)=0$. This can be done effectively exactly because we are looking for $\Pi_{1}^{0}$ indices, and the search is uniform. We then let $i_{s}$ be the least such that $\mathcal{C}_{n}\left(i_{s}\right)$ is undefined, and take $\mathcal{C}_{n}\left(i_{s}+\ell\right)=e_{t, \ell}$ for each $\ell<2^{t}$. We also set $G_{t, s}=G_{t, s-1} \cup k$.

Now for each $t$ with $f(t)=1$, there will be some $s$ such that $f_{s^{\prime}}(t)=f_{s}(t)=1$ for all $s^{\prime}>s$. Then at stage $s$ we have added to $\mathcal{C}_{n}$ the $\Pi_{1}^{0}$ indices $\left\{e_{t, i}: i<2^{t}\right\}$ guaranteeing that $\left\{\pi_{t, k, j}: j<t\right\}$ is shattered for some $k$. 
For each $t$ such that $f(t)=0$ and each $s$ such that $f_{s}(t)=1$, there is some later stage $s^{\prime}$ such that $f_{s^{\prime}}(t)=0$, so any indices added at stage $s$ will be indices for a tree with no paths - that is, for the empty concept.

Note that if the same $t$ receives attention infinitely often - that is, if infinitely many different sets of classes are added to $\mathcal{C}_{n}$ to guarantee that the VC dimension of $\mathcal{C}_{n}$ is at least $t$, this does not inflate the $\mathrm{VC}$ dimension beyond $t$. Indeed, the sets of witnesses will be pairwise disjoint, so no concept in $\mathcal{C}_{n}$ will include any mixture of witnesses from different treatments; the resulting sets will not be shattered.

We further note that all the $\Pi_{1}^{0}$ classes in $\mathcal{C}_{n}$ are computable. Indeed, each $c \in \mathcal{C}_{n}$ consists of finitely many (perhaps no) computable paths. Thus, $\mathbb{C}_{n}$ is an effective concept class.

Now if $n \notin S$, then $f(s)=1$ for at most finitely many $s$, so that the $\mathrm{VC}$ dimension of $\mathcal{C}_{n}$ is finite. If $n \in S$, then $f(s)=1$ for infinitely many $s$, so that the VC dimension of $\mathcal{C}_{n}$ is infinite (since sets of arbitrarily large size will be shattered).

\section{REFERENCES}

1. A. Beros, Learning theory in the arithmetic hierarchy, preprint, 2013.

2. C. M. Bishop, Pattern recognition and machine learning, Information Science and Statistics, Springer, 2006.

3. A. Blumer, A. Ehrenfeucht, D. Haussler, and M. K. Warmuth, Learnability and the VapnikChervonenkis dimension, Journal of the ACM 36 (1989), 929-965.

4. M. Braverman and M. Yampolsky, Computability of julia sets, Algorithms and Computation in Mathematics, no. 23, Springer, 2009.

5. W. Calvert, The isomorphism problem for computable Abelian p-groups of bounded length, Journal of Symbolic Logic 70 (2005), 331-345.

6. W. Calvert, D. Cenzer, V. Harizanov, and A. Morozov, Effective categoricity of equivalence structures, Annals of Pure and Applied Logic 141 (2006), 61-78.

7. $-\Delta_{2}^{0}$-categoricity of Abelian p-groups, Annals of Pure and Applied Logic 159 (2009), 187-197.

8. W. Calvert, V. Harizanov, J. F. Knight, and S. Miller, Index sets of computable structures, Algebra and Logic 45 (2006), 306-325.

9. D. Cenzer, $\Pi_{1}^{0}$ classes in computability theory, Handbook of Computability, Studies in Logic and the Foundations of Mathematics, no. 140, Elsevier, 1999, pp. 37-85.

10. D. Cenzer and J. Remmel, Index sets for $\Pi_{1}^{0}$ classes, Annals of Pure and Applied Logic 93 (1998), 3-61

11. M. Friend, N. B. Goethe, and V. Harizanov, Induction, algorithmic learning theory, and philosophy, Logic, Epistemology, and the Unity of Science, vol. 9, Springer, 2007.

12. E. M. Gold, Language identification in the limit, Information and Control 10 (1967), 447-474.

13. S. S. Goncharov and J. F. Knight, Computable structure and non-structure theorems, Algebra and Logic 41 (2002), 351-373.

14. V. Harizanov and F. Stephan, On the learnability of vector spaces, Journal of Computer and System Sciences 73 (2007), 109-122.

15. M. J. Kearns and U. V. Vazirani, An introduction to computational learning theory, MIT Press, 1994.

16. A. G. Melnikov and A. Nies, The classification problem for compact computable metric spaces, The Nature of Computation: Logic, Algorithms, Applications, Lecture Notes in Computer Science, vol. 7921, Springer, 2013, pp. 320-328.

17. S. Russell and P. Norvig, Artificial intelligence, 3rd ed., Prentice Hall, 2010.

18. R. I. Soare, Recursively enumerable sets and degrees, Springer-Verlag, 1987.

19. F. Stephan and Y. Ventsov, Learning algebraic structures from text, Theoretical Computer Science 268 (2001), 221-273.

20. L. G. Valiant, A theory of the learnable, Communications of the ACM 27 (1984), 1134-1142.

21. V. N. Vapnik and A. Ya. Chervonenkis, On the uniform convergence of relative frequencies of events to their probabilities, Theory of probability and its applications 16 (1971), 264-280.

22. K. Weihrauch, Computable analysis, Texts in Theoretical Computer Science, Springer, 2000. 
Department of Mathematics, Mail Code 4408, Southern Illinois University, CarbonDale, 1245 Lincoln Drive, Carbondale, Illinois 62901

E-mail address: wcalvert@siu.edu 